\title{
A pragmatist's guide to the assessment of decision-making capacity
}

\author{
Rocksheng Zhong, Dominic A. Sisti and Jason H. Karlawish
}

\section{Summary}

Choice, understanding, appreciation and reasoning compose the standard model of decision-making capacity. Difficulties in determining capacity can arise when patients exhibit partial impairment. We suggest that a pragmatic approach, focusing on how capacity status affects the ultimate decision to override the patient's wishes, can help evaluators resolve difficult cases.

\section{Declaration of interest}

None.

\section{Keywords}

Consent and capacity; ethics; psychiatry and law.

\section{Copyright and usage}

(c) The Royal College of Psychiatrists 2019
Rocksheng Zhong is a lecturer in the Department of Psychiatry at the Yale School of Medicine in Connecticut, USA. Dominic A. Sisti is an assistant professor in the Department of Medical Ethics and Health Policy, as well as Director of the Scattergood Program for Applied Ethics of Behavioral Health Care, at the Perelman School of Medicine at the University of Pennsylvania, USA. Jason H. Karlawish is a professor in the Departments of Medicine, Medical Ethics and Health Policy, and Neurology, as well as Co-Director of the Penn Memory Center, at the Perelman School of Medicine at the University of Pennsylvania, USA.

\section{The standard model of decision-making capacity}

Decision-making capacity is an essential element of informed consent, and therefore the ethical practice of medicine. ${ }^{1}$ The current standard of care uses the Appelbaum and Grisso four abilities model. ${ }^{2,3}$ Clinicians evaluate patients' abilities to express a choice, understand, appreciate and reason against a 'sliding scale', such that the 'stringency of the test applied varies directly with the seriousness of the likely consequences of patients' decisions'. ${ }^{2}$ Although many capacity evaluations are straightforward and uncontroversial, occasional grey-area cases can provoke uncertainty and moral distress among clinicians. This essay provides a pragmatic approach for a particular kind of case, in which the capacity determination may result in a clinician overriding a patient's expressed wishes. We argue that attention to the issue of whether to respect a patient's choice can help resolve apparent conflicts and simplify the evaluator's task.

Appelbaum and Grisso formulate four decisional abilities. ${ }^{1}$ Choice is the ability to communicate a clear and consistent choice. Understanding is the ability to grasp the meaning of relevant information. Appreciation is the ability to acknowledge the medical situation and likely consequences of interventions. Reasoning is the ability to rationally manipulate relevant information by weighing options and consequences. The degree of sophistication to which a patient must demonstrate these abilities depends on the seriousness of the expected consequences. ${ }^{2}$ As a decision's risk of harm escalates, a patient must meet progressively higher bars of decisional ability. For instance, a choice to take a multivitamin is low risk and requires only a low degree of decisional ability, whereas a choice to undergo experimental chemotherapy is high risk and requires a high degree of decisional ability.

\section{Partial impairment}

Assessments of decision-making capacity are often straightforward. Some patients clearly lack capacity; for example, a patient with delirium who can only mumble incoherently demonstrates no ability to express a choice, understand, appreciate or reason. On the opposite end of the spectrum, most people in out-patient settings who do not have significantly compromising illnesses have no decisional impairment.

The difficult cases arise in the grey area, where a partially impaired individual makes a moderately risky decision. One such scenario is a patient with a history of bipolar disorder who declines recommended hospital care, such as intravenous antibiotics for a serious infection. They confidently state that although they are aware of, and accept, the risks, they believe they can beat the odds because they have escaped worse situations unharmed. Additional history-taking reveals that the patient has been sleeping 5 hours nightly and feels energised. Their speech is fast, but not obviously pressured. Such a person expresses a clear choice (no further inpatient treatment) and says that they understand the negative health outcomes associated with declining treatment. However, clinicians may disagree about whether the patient shows adequate appreciation and reasoning. Some might argue that the patient's acceptance of consequences and ability to articulate a reason (prior life experience) are sufficient. Others might argue that the patient's appreciation and reasoning are impaired by burgeoning hypomania. Put another way, this person has some capacity, but do they have enough?

Experts observe that requests for capacity assessment often obscure underlying interpersonal or communication issues. ${ }^{4}$ They advise evaluators, when possible, to address those underlying issues rather than focusing strictly on the capacity question. ${ }^{5}$ Patients may have unattended needs, such as pain or other discomfort, financial concerns or insufficient knowledge about their condition, that provoke conflict and capacity questions. ${ }^{6}$ Some patients require additional time to process new information. Others have decisional impairments that improve as their illness resolves, and others are amenable to persuasion or negotiation. ${ }^{7}$ The consultation-liaison psychiatry literature describes a multitude of such scenarios and strategies to manage them.

\section{Practical decision-making: whether to treat over objection}

Although the above approaches are often effective, a subset of capacity assessment requests will remain. For such cases, we suggest that a focus on the practical outcomes of the assessment may clarify the relevant stakeholders' positions and, in turn, simplify the capacity assessment. 
Clinicians usually assess a patient's decision-making capacity not for its own sake, but to answer a question: Should we respect the patient's wishes? A person with capacity may refuse even lifesaving treatment; a person lacking capacity may be overruled. ${ }^{1}$ When someone declines recommended treatment, that individual's capacity status is an essential consideration in determining whether to treat over objection. However, if capacity is unclear, an alternative inquiry can help clinicians decide whether to respect a patient's wishes or proceed with forced treatment: is the treatment, when involuntarily administered, medically appropriate and available?

Returning to the earlier example, suppose that a patient with a history of bipolar disorder who may be hypomanic declines intravenous antibiotics in favour of discharge. Some patients will acquiesce to strong enough pressure and will cooperate if faced with the possibility of forced treatment. However, if the patient persists in their choice, their capacity status will matter only if the treatment team is willing to proceed with treatment over objection, which may include involuntary medications, physical restraints, detention in the hospital and constant observation. If the team does not offer these treatment modalities, then the patient's capacity is, in some sense, moot. If they have capacity, then they are free to decline treatment. If they lack capacity, the treatment team will not treat them involuntarily in the hospital anyway. Either way, the patient avoids treatment and is discharged.

Thus, capacity evaluators should collaborate closely with treatment providers to determine at what point the balance of risks and benefits shifts from honouring the patient's wishes to forcing treatment. Furthermore, clinicians should also factor in the risks of involuntary treatment. The use of restraints, for instance, has been associated with direct injury, pressure sores, prolonged length of hospital stay and death. ${ }^{8}$ When risks clearly outweigh benefits, then the intervention is medically inappropriate. For example, no one would suggest that a patient be restrained and forced to consume a multivitamin. However, intravenous antibiotics may be beneficial depending on the severity of the patient's infection. On the one hand, if the patient were septic and at imminent risk of death, then the treatment team may conclude that forced treatment is nevertheless indicated. On the other hand, if they were haemodynamically stable and able to mitigate their risk in other ways, such as oral medications and close out-patient follow-up, then involuntary intravenous antibiotics in the hospital may not be appropriate. In the latter case, clinicians may exclude involuntary hospital treatment as not medically indicated, and therefore unavailable.

In short, capacity is only practically important when the treatment team is willing to proceed with forced treatment. Absent this condition, the outcome would be the same as simply honouring the patient's choice, and there is no need (apart from intellectual satisfaction) to assess capacity. Thus, an otherwise difficult question (does a partially impaired individual have enough capacity?) gives way to a simpler question: does it matter whether this individual has capacity?

\section{Critiques}

It is arguable that this approach puts the cart before the horse. Consideration of treatment over objection presupposes a finding of incapacity. This position, however, does not recognise the fact that physicians implicitly consider the modality and consequences of interventions when making all manner of clinical decisions. Indeed, evaluation of a clinical determination with an eye toward process and outcomes is a well-described form of clinical judgement and practical wisdom. ${ }^{9,10}$
Furthermore, in keeping with the pragmatic approach of this paper, the scenarios and thought processes mentioned here are consistent with the lived experience of medicine. Clinicians do not assess capacity in a vacuum. They are almost always aware of treatment teams' planning and expectations.

\section{Conclusion}

Assessments of decision-making capacity can be interpersonally and morally fraught. In this paper, we highlight an opportunity for clinicians to make implicit assumptions explicit and, in doing so, simplify particularly challenging cases involving capacity evaluation. By taking a pragmatic approach and anticipating the outcomes of a capacity determination, evaluators can transform a difficult question into an easier one. If no clinician would treat over the patient's objection, then whether the patient has capacity is immaterial to the question of what to do next. This sidestepping of the capacity question may be intellectually unsatisfying but is similar to the consultation-liaison psychiatry strategies advocating that practitioners address underlying issues. When all parties realise that the prerequisites for opposing the patient's choice are not met, the conflict between the patient and treating physicians may melt away.

Rocksheng Zhong, MD, MHS (D), Lecturer, Department of Psychiatry, Yale School o Medicine, USA; Dominic A. Sisti, PhD, Assistant Professor, Department of Medical Ethics and Health Policy, University of Pennsylvania; and Director of the Scattergood Program for Applied Ethics of Behavioral Health Care, Perelman School of Medicine, University of Pennsylvania, USA; Jason $\mathbf{H}$. Karlawish, MD, Professor, Departments of Medicine Medical Ethics and Health Policy, and Neurology, University of Pennsylvania; and Co-Director of the Penn Memory Center, Perelman School of Medicine, University of Pennsylvania, USA

Correspondence: Rocksheng Zhong, 3900 Montclair Road, Floor 1 \#131272,

Birmingham, AL 35213, USA. Email: rocksheng.zhong@yale.edu

First received 10 Feb 2018, accepted 19 Dec 2018

\section{Funding}

This work was supported by the University of Pennsylvania, Department of Psychiatry Clinical Research Scholars Program, as well as grants from the National Institute on Aging (P30AG010124) and the Centers for Disease Control Health Brain Research Network (U48 DP AG010124) and the Centers for Disease Contro

\section{Acknowledgements}

We would like to thank Henry Bleier (MD), Behdad Bozorgnia (MD), John Northrop (MD, PhD), Justin Piershalski (MD), James Stinnett (MD), and the Consultation-Liaison Psychiatry service at the Hospital of the University of Pennsylvania for their comments and assistance throughout the writing and revision process.

\section{References}

1 Grisso T, Appelbaum PS. Assessing Competence to Consent to Treatment: A Guide For Physicians and Other Health Professionals. Oxford University Press, 1998.

2 Appelbaum PS. Assessment of patients' competence to consent to treatment N Engl J Med 2007; 357(18): 1834-40.

3 Moye J, Marson DC. Assessment of decision-making capacity in older adults: An emerging area of practice and research. J Gerontol B Psychol Sci Soc Sci 2007; 62(1): P3-11.

4 Umapathy C, Ramchandani D, Lamdan RM, Kishel LA, Schindler BA Competency evaluations on the consultation-liaison service. Some overt and covert aspects. Psychosomatics 1999; 40(1): 28-33.

$5 \mathrm{Kim}$ SYH. Evaluation of Capacity to Consent to Treatment and Research. Oxford University Press, 2010.

6 Kontos N, Querques J, Freudenreich O. Capable of more: some underemphasized aspects of capacity assessment. Psychosomatics 2015; 56(3): 217-26. 
7 Siegel AM, Bleier HR. The role of negotiation in consultation-liaison psychiatry. Psychosomatics 2017; 58(2): 187-90.

8 Evans D, Wood J, Lambert L. Patient injury and physical restraint devices: a systematic review. J Adv Nurs 2003; 41(3): 274-82.
9 Pellegrino ED. The anatomy of clinical judgments. In Clinical Judgment: A Critical Appraisal (eds Engelhardt HT, Spicker SF, Towers B): 169-94: Springer, 1979.

10 Henry SG. Recognizing tacit knowledge in medical epistemology. Theor Med Bioeth 2006; 27(3): 187-213. 